\title{
Walras Equilibrium with Coordination
}

Grodal, Birgit; Vind, Karl

Publication date:

2000

Document version

Early version, also known as pre-print

Citation for published version (APA):

Grodal, B., \& Vind, K. (2000). Walras Equilibrium with Coordination. Department of Economics, University of Copenhagen. 


\title{
DISCUSSION PAPERS Department of Economics University of Copenhagen
}

$$
00-18
$$

Walras Equilibrium with Coordination

\author{
Birgit Grodal \\ Karl Vind
}

Studiestræde 6, DK-1455 Copenhagen K., Denmark

Tel. +45 35323082 - Fax +45 35323000

http://www.econ.ku.dk 


\title{
Walras Equilibrium with Coordination
}

\author{
Birgit Grodal $^{*} \quad$ Karl Vind ${ }^{\dagger}$
}

September 2000

\begin{abstract}
We consider a pure exchange economy with private ownership in which consumers have interdependent preferences. Hence, consumers' preferences are defined on the states of the economy. In a Walras equilibrium for such an economy, it may, of course, be possible for two or more consumers to simultaneously change their net trades and thereby obtain a preferred state. We use the concept of coordination introduced by Vind (1983) to define an exogenously given coordination structure in the economy and define a new equilibrium concept, Walras equilibrium with coordination. In such an equilibrium individual consumers take prices and initial endowments as given, and consumers do not expect to be able to obtain a preferred state when they coordinate their choice of net trades. By using the existence theorem for an equilibrium in a social system with coordination, we set conditions for the existence of a Walras equilibrium with coordination.
\end{abstract}

Keywords: Walras equilibrium with coordination, coordination, social system, households, incomplete markets.

JEL Classification: D50, D62, D11.

\footnotetext{
${ }^{*} Ø$ konomisk Institut, Københavns Universitet, Studiestræde 6, DK-1455 København, Denmark

${ }_{\dagger} \varnothing$ konomisk Institut, Københavns Universitet, Studiestræde 6, DK-1455 København, Denmark
} 


\section{Introduction}

We consider a pure exchange economy with private ownership in which consumers have interdependent preferences. We assume that a state of the economy specifies the net trade of each consumer and that consumers' preferences are defined on the states of the economy. In a Walras equilibrium for such an economy, it may, of course, be possible for two or more consumers to simultaneously change their net trades and thereby obtain a preferred state. In order to allow consumers to coordinate their net trades, we use the concept of coordination introduced by Vind (1983) to define an exogenously given coordination structure in the economy and to define a new equilibrium concept, Walras equilibrium with coordination.

Coordination is specified by coordination functions for the consumers and an approval function. A coordination function for a consumer specifies for each state $x$ of the economy and each alternative state $y$ that state the consumer believes will be the outcome if in state $x$ all consumers $c \in C$ change their net trades to $y(c)$. Clearly, a coordination function could specify that the consumer only coordinates with himself or, at the other extreme, that he coordinates with all other consumers. Typically, each consumer coordinates his net trade with a subset of the set of consumers. We do not assume that coordination is symmetric, that is, consumer $c$ may coordinate with $d$ without $d$ coordinating with $c$. Moreover, two consumers may coordinate in some commodities and not in others.

In traditional economic theory a household is endowed with a vector of initial resources and a preference relation which is often assumed to be a total preorder. However, a household typically has several members. In our formalization of a household, each member will be endowed with his own initial resources and his own preference relation. Moreover, each member is described by a coordination function which may, for example, specify that he coordinates his net trade with the net trades of all the other members of the household. As we do not allow members of a household to transfer budgets, our approach leads to a household decision problem that is different from the one discussed in Browning and Chiappori (1998) and Gersbach and Haller (1999), for example.

Clearly, to define an equilibrium state, we also have to specify the set of consumers who has to approve a change from one state $x$ to another state $y$. In the traditional Walras equilibrium, where a consumer (household with a single budget constraint) only considers his own change of net trade, the change should just be approved by the consumers who change their net trades; hence, as there is no coordination, it is enough to look at a deviation by a single consumer (household). In our formalization, we explicitly have to define the set of consumers who has to approve a change. This is done by enlarging the description of the economy with an approval function. For each state $x$ and each alternative state $y$, this function specifies the set of consumers who has to approve a change from $x$ to $y$. Note that, since consumers have different coordination functions, they expect 
different states to be the outcome of the change from $x$ to $y$. In the household example the approval function might, for example, specify that the change has to be approved by all those consumers who are members of a household where at least one consumer has changed his net trade.

The market institution we study in this paper is a complete Walrasian market. Thus, all commodities have associated prices. We do not allow consumers to transfer budgets even if they coordinate with other consumers. Hence, a consumer has to choose a net trade with non-positive value at the prevailing prices. In a Walras equilibrium with coordination, consumers take prices and initial endowments as given, satisfy their budget constraint, and there is market clearing. Moreover, there does not exist an alternative state y such that each of the consumers who has to approve the change to y satisfies his budget constraint and prefers the state which he believes will be the outcome from the change to y.

By using the existence theorem for an equilibrium in a social system with coordination (Vind 1983 and Keiding 1985), we set conditions for the existence of a Walras equilibrium with coordination. Apart from the assumptions on the coordination functions and the approval function, the conditions correspond to those used for the existence of a Walras equilibrium in an economy with externalities by Shafer and Sonnenschein (1975). In particular, we do not assume that preferences are complete or transitive.

The concept of a Walras equilibrium with coordination and the existence result are interesting not only in terms of the most obvious interpretation, namely to analyze equilibria in an economy with externalities in consumption. It also turns out that Walras equilibrium with coordination in partitions is a useful tool for analyzing economies where consumers have several budget constraints ${ }^{1}$. By splitting each of the consumers into as many agents as he has budget constraints, we obtain a new economy in which each agent has only one budget constraint, but agents have interdependent preferences even if consumers have independent preferences. In order to reach an equivalence between the original maximization problem for a consumer and the maximization problems for the agents corresponding to him in the new economy, we assume that agents coming from the same consumer coordinate their actions. This tool is used in Grodal and Vind (1989) to study economies with a much richer set of market institutions.

In an economy where all consumers have independent preferences there is no need for coordination, and the definition of a Walras equilibrium with coordination reduces to the definition of a Walras equilibrium. Similarly, if each consumer only coordinates his choice of net trade with himself a Walras equilibrium with coordination is a usual Walras equilibrium. In general the set of Walras equilibria with coordination depends on the coordination functions as well as the

\footnotetext{
${ }^{1}$ When markets are incomplete consumers have several budget constraints; see for example Duffie and Shafer (1985).
} 
approval function. Walras equilibria with coordination will not, in general, be Pareto optimal even if all consumers coordinate with all other consumers in all commodities. The reason is that, although the coordination functions allow consumers to coordinate their net trades, consumers have to satisfy their budget constraints individually.

In section 2, we define the economy. Section 3 contains the equilibrium definition. The existence theorem is presented in section 4 . Section 5 contains some examples of coordination structures.

\section{The economy}

We consider a pure exchange economy

$$
\mathcal{E}=\left(L, C, Y,\left(X_{c}, P_{c}, e_{c}\right)_{c \in C}, g\right)
$$

where for all $c \in C$

(i) $L$ is a finite set of commodities,

(ii) $C$ is a finite set of consumers,

(iii) $Y=\mathbb{R}^{C L}$ is the state space of the economy,

(iv) $X_{c} \subset Y$ is the set of net trade states that are feasible for consumer $c$,

(v) $\mathcal{P}_{c}: X_{c} \rightarrow 2^{X_{c}}$ is the preference correspondence for consumer $c$,

(vi) $e_{c}: Y \times Y \rightarrow Y$ is the coordination function for consumer $c$,

(vii) $g: Y \times Y \rightarrow 2^{C}$ is the approval function.

A state $x \in Y$ specifies the net trade $x(c) \in \mathbb{R}^{L}$ for each consumer $c \in$ $C$. We use the notation $x(c, h)$ for consumer $c$ 's net trade in commodity $h \in$ $L$. Apart from the coordination functions $e_{c}, c \in C$ and the approval function $g$, the description of the economy $\mathcal{E}$ is the standard one when consumers have interdependent preferences: Each consumer $c$ is described by the states $X_{c}$ which are feasible for him, and a preference correspondence $\mathcal{P}_{c}$ where $\mathcal{P}_{c}(x)$ are the states preferred by consumer $c$ to the state $x$. The coordination function $e_{c}$ specifies for each state $x \in Y$ and each alternative state $y \in Y$ the state $e_{c}(x, y)$ which consumer $c$ expects to be the outcome when, in state $x$, the consumer $d$ has chosen the net trade $y(d), d \in C$. Moreover, we assume that there is an approval function $g: Y \times Y \rightarrow 2^{C}$. For each pair of states $x, y \in Y, g(x, y) \subset C$ gives the set of consumers who has to approve the change from $x$ to $y$. 
We will assume that the coordination functions satisfy the following assumption:

Assumption (Coordination Assumption). For each consumer $c \in C$ the coordination function $e_{c}: Y \times Y \rightarrow Y$ satisfies

(i) For all $(d, h) \in C \times L: e_{c}(x, y)(d, h)=\left\{\begin{array}{cc}x(d, h) & \text { for all } x, y \in Y \\ \text { or } & \\ y(d, h) & \text { for all } x, y \in Y\end{array}\right.$

(ii) $e_{c}(x, y)(c)=y(c)$ for all $x, y \in Y$

The assumption specifies the special coordination functions we use in this paper. The first part of the assumption states that consumer $c \in C$ expects states where consumer $d$ 's net trade in commodity $h$ is $d$ 's old net trade in $h$ for all states $x$ and alternative states $y$, or it is $d$ 's new net trade in commodity $h$. We say that $c$ coordinates his net trade with the net trade in commodity $h$ of consumer $d$ if $e_{c}(x, y)(d, h)=y(d, h)$ for all $x, y \in Y$. We say that $c$ coordinates with consumer $d$ if there exists a commodity $h$ such that $c$ coordinates his net trade with $d$ 's net trade in commodity $h$. The second part of the assumption states the natural condition that consumer $c$ coordinates his net trade in all commodities with himself. It should be noted that we do not assume that the coordination relation is symmetric or transitive.

\section{Assumption (Feasible Net Trade Assumption).}

(A) For all $c \in C$ the feasible set $X_{c}$ and the coordination function $e_{c}$ satisfy the following: for all $x \in \cap_{d \in C} X_{d}$ and $y \in Y, e_{c}(x, y) \in X_{c} \Rightarrow y \in X_{c}$.

(B) The feasible sets $X_{c}, c \in C$ and the coordination functions $e_{c}, c \in C$ satisfy the following: for all $x, y \in \cap_{c \in C} X_{c}, e_{d}(x, y) \in \cap_{c \in C} X_{c}$ for all $d \in C$.

Assume that, if a consumer $c \in C$ coordinates with consumer $d$, he coordinates with $d$ in all commodities $h \in L$. The feasible net trade assumption will then be satisfied if, for all $c \in C$, the feasible set $X_{c}$ has the form $X_{c}=X_{c}^{\prime} \times \prod_{d \neq c} \mathbb{R}^{L}$ for some $X_{c}^{\prime} \subset \mathbb{R}^{L}$. That is, a state $x$ is feasible for $c$ iff $c^{\prime}$ s net trade is in $X_{c}^{\prime}$. The assumption is also satisfied in the following case: Let $R_{c}$ be the set of consumers with whom $c$ coordinates and assume that there exist individually feasible sets of net trades $X_{c}^{\prime} \subset \mathbb{R}^{L}$ for $c \in C$ such that $X_{c}=\prod_{d \in R_{c}} X_{d}^{\prime} \times \prod_{d \notin R_{c}} \mathbb{R}^{L}$. This last assumption says that a state is feasible for consumer $c$ iff all the consumers with whom he coordinates have net trades in the individually feasible sets of net trades.

As mentioned above, the approval function $g: Y \times Y \rightarrow 2^{C}$ specifies the set of consumers who has to approve the change from state $x$ to state $y$. An approval function $g$ will naturally have the following property: $\{c \in C \mid x(c) \neq y(c)\} \subset$ 
$g(x, y) \subset\left\{c \in C \mid e_{c}(x, y) \neq x\right\}$ for all $x, y \in Y$. This property amounts to the following: First, all consumers who actually change their net trade should approve the change. Moreover, only consumers who coordinate with a consumer who has changed his net trade are included in the set of consumers who has to approve the change. In the existence theorem in section 5, we assume that $g(x, y)=\left\{c \in C \mid e_{c}(x, y) \neq x\right\}$ for all $x, y \in Y$, that is, all consumers who coordinate with some consumer who changes his net trade have to approve the change.

\section{Walras Equilibrium with Coordination}

We shall use the following notation. Let 1 be the vector $(1, \cdots, 1) \in \mathbb{R}^{C L}$. Moreover, let $\Delta=\left\{p \in \mathbb{R}_{+}^{L} \mid \sum_{h=1}^{L} p_{h}=1\right\}$ be the non-negative price simplex in $\mathbb{R}^{L}$.

The definition of feasible states for our economy $\mathcal{E}$ is:

Definition (Feasible States). A state $x \in Y$ is feasible for the economy $\mathcal{E}$ if

(i) $x \in \cap_{c \in C} X_{c}$ and

(ii) $\sum_{c \in C} x(c) \leq 0$

The set of feasible states is denoted $F(\mathcal{E}) \subset Y$.

Lemma . Assume that $\left\{x(c) \in \mathbb{R}^{L} \mid x \in X_{c}\right\}$ is bounded from below for each consumer $c \in C$. Then there exists $K>0$ such that $F(\mathcal{E}) \subset$ int $\widetilde{Y}$ where $\widetilde{Y}=$ $\{x \in Y \mid-K \mathbf{1} \leq x \leq K \mathbf{1}\}$.

Proof. By assumption $\cap_{c \in C} X_{c}$ is bounded below. As, moreover, $\sum_{c \in C} x(c) \leq 0$ for all $x \in F(\mathcal{E})$ it follows that $F(\mathcal{E})$ is bounded from above.

Definition (Walras Equilibrium with Coordination). A Walras equilibrium with coordination in the economy $\mathcal{E}$ is a pair $(p, x) \in \Delta \times F(\mathcal{E})$ consisting of a price and a feasible state such that

(I) $p x(c) \leq 0$ for all $c \in C$,

(II) there does not exist $y \in \mathbb{R}^{C L}$ with $y \neq x$, such that for all $c \in g(x, y)$, $p y(c) \leq 0$ and $e_{c}(x, y) \in \mathcal{P}_{c}(x)$,

(III) $p_{h} \sum_{c \in C} x_{h}(c)=0$ for all $h \in L$.

The equilibrium condition (I) states that all consumers take prices as given and choose a net trade with non-positive value at the given prices. Hence, no transfer of budget is allowed even if consumers coordinate their net trades. The 
equilibrium (II) condition states that there does not exist a non-empty set of consumers who can simultaneously change their net trades such that all consumers who have to approve the change expect to obtain a preferred state. The condition (III) states that the price of a commodity is zero if the aggregate net trade is negative.

Clearly, if the feasible states and the preferences of a consumer are independent (i.e., feasibility and the preferences of a consumer are defined by his own net trades), then for any coordination functions, a Walras equilibrium with coordination is exactly the same as a Walras equilibrium in the economy where the coordination functions have been disregarded.

\section{The existence theorem}

Before stating our existence theorem for the economy $\mathcal{E}$, we introduce the assumption of local non-satiation in $\mathcal{E}$.

Assumption (Local Non-Satiation). For all $x \in X$ and for all $c \in C$ and all open neighborhoods $U$ in $\mathbb{R}^{L}$ of $x(c)$ there exists $x^{\prime} \in U$ such that letting the state $y \in Y$ be defined by $y(d)=x(d)$ for $d \neq c$ and $y(c)=x^{\prime}$ then $e_{d}(x, y) \in \mathcal{P}_{d}(x)$ for all $d$ with $e_{d}(x, y) \neq x$. If the assumption is satisfied for consumer $c$, we say that $c$ is locally non-satiated.

The condition states that consumer $c$ can find a net trade $x^{\prime}$ locally such that he, as well as the consumers that coordinate with him, get a preferred state if $c$ changes his action from $x(c)$ to $x^{\prime}$. Clearly, the condition is satisfied if all consumers who coordinate with a given consumer are benevolent and each consumer has monotone preferences or just local non-satiation in his own consumption.

Theorem 1 (Equilibrium). Let $\mathcal{E}$ be the economy as described above and assume

(i) $X_{c}$ is closed, convex, $0 \in$ int $X_{c}$, and $\left\{x(c) \in \mathbb{R}^{L} \mid x \in X_{c}\right\}$ is bounded from below, for all $c \in C$,

(ii) $\mathcal{P}_{c}$ is irreflexive and has open graph and convex values, for all $c \in C$,

(iii) all consumers $c \in C$ are locally non-satiated,

(iv) the coordination assumption is satisfied,

(v) the feasible net trade assumption is satisfied,

(vi) for all $c \in C$ there exists $\left(X^{c}, \mathcal{P}^{c}\right)$ such that $X_{c} \subset$ int $X^{c}$ and $\mathcal{P}_{c}(x)=$ $\mathcal{P}^{c}(x) \cap X_{c}$ for all $x \in X_{c}$, and $\left(X^{c}, \mathcal{P}^{c}\right)$ satisfies (i) and (ii), 
(vii) $g(x, y)=\left\{c \in C \mid e_{c}(x, y) \neq x\right\}$ for all $x, y \in Y$.

Then there exists a Walras equilibrium with coordination in $\mathcal{E}$.

It is well known that the existence of a Walras equilibrium in an economy can be shown by converting the economy into a social system by adding an agent who sets prices, using an existence theorem for a Nash equilibrium in the social system, and finally showing that a Nash equilibrium in the social system is a Walras equilibrium in the original economy (see Arrow and Debreu 1954 for a proof). Our proof of the existence of a Walras equilibrium with coordination parallels this proof. However, we convert our economy with coordination into a social system with coordination as defined by Vind (1983) and use the existence theorem for an equilibrium in a social system with coordination by Keiding (1985). The definition of a social system with coordination and the equilibrium existence theorem are included in the appendix.

Proof. First, we truncate the economy $\mathcal{E}$. By the lemma there exists a compact and convex set $\widetilde{Y}=\{x \in Y \mid-K \mathbf{1} \leq x \leq K \mathbf{1}\}$ with $K>0$ such that $F(\mathcal{E}) \subset$ int $\widetilde{X}$. Let $\widetilde{X}_{c}=X_{c} \cap \widetilde{Y}$ and let $\tilde{\mathcal{E}}$ be the corresponding truncated economy. Second, we define the social system with coordination $\Gamma=\left(A,\left(X_{a}, \beta_{a}, \mathcal{P}_{a}, e_{a}\right)_{a \in A}, g\right)$ corresponding to the economy $\tilde{\mathcal{E}}$. The agents $A$ are obtained by adding an agent $a=0$ who sets prices, that is, $A=C \cup\{0\}$. The state space for $\Gamma$ is $y=\mathbb{R}^{L} \times \mathbb{R}^{C L}$. The feasible states for agent $a \in C$ are defined by $\mathcal{X}_{a}=\mathbb{R}^{L} \times \widetilde{X}_{a}$ and for $a=0$ by $X_{0}=\Delta \times \mathbb{R}^{C L}$. Let $X=\cap_{a \in A} X_{a}$. The constraint function $\beta_{a}: X \rightarrow X$ is defined by $\beta_{a}(p, x)=\{(q, y) \in \mathcal{X} \mid p y(a) \leq 0\}$ for $a \in C$. For $a=0$ we let $\beta_{0}(p, x)=X$. For all agents $a \in C$ and all $((p, x)(q, y)) \in X \times X$ we let $e_{a}((p, x),(q, y))=\left(p, e_{a}(x, y)\right)$, that is, all consumers use their coordination functions from $\mathcal{E}$ and do not coordinate with the price maker ${ }^{2}$. Note that the definition of $\widetilde{Y}$ together with the assumption (v) imply that $e_{a}((p, x),(q, y)) \in \mathcal{X}$ for all $a \in C$. For $a=0$ and all $((p, x)(q, y)) \in \mathcal{X} \times \mathcal{X}$ we let $e_{0}((p, x),(q, y))=(q, x) \in \mathcal{X}$. That is, the price maker does not coordinate with any of the consumers. Similarly, the preferences of agent $a \in C$ are defined by $\mathcal{P}_{a}(p, x)=\{(q, y) \in \mathcal{X}$ $\left.y \in \mathcal{P}_{a}(x)\right\}$ for all $(p, x) \in \mathcal{X}$. The preferences of the price setter are defined in the usual way by $\mathcal{P}_{0}(p, x)=\left\{(q, y) \in X \mid q \cdot \sum_{c \in C} x(c)>p \cdot \sum_{c \in C} x(c)\right\}$. Finally, the approval function $g$ in $\Gamma$ is defined in the same way as in $\mathcal{E}$, that is, $g((p, x)(q, y))=\left\{a \in A \mid e_{a}((p, x),(q, y)) \neq(p, x)\right\}$.

In order to obtain an equilibrium in our social system with coordination, we have to show that the assumptions of Theorem 2 in the Appendix are satisfied. By way of construction we have that $\widetilde{X} \subset \mathbb{R}^{L+C L}$ is non-empty, compact, and convex. Thus assumption (a) is satisfied. By definition, $\beta: X \times X \rightarrow X$ is given by $\beta(p, x)=\cap_{a \in A} \beta_{a}(p, x)=\{(q, y) \in X \mid p \cdot x(c) \leq 0$ for all $c \in C\}$.

\footnotetext{
${ }^{2}$ For notational simplicity, we use the same symbols for the coordination functions of the consumers and the approval function in $\Gamma$ and in $\mathcal{E}$.
} 
Clearly, $\beta$ has closed and convex values. By assumption (i) the values are nonempty. Also, the correspondence $\beta$ is upper hemi continuous. To see that it is lower hemi continuous, consider $(p, x) \in \mathcal{X},(q, y) \in \beta(p, x)$, and a sequence $\left(p^{n}, x^{n}\right) \in \mathcal{X}$ converging to $(p, x)$. By assumption (i) and the construction of $\widetilde{Y}$, there exists $\underline{u}<<0 \in \widetilde{X}$. Hence, for all $n$ we have $p^{n} \underline{u}(c)<0$ for all $c \in C$. We now construct a sequence $y^{n}$ converging to $y$ with $\left(q, y^{n}\right) \in \cap_{c \in C} \beta_{c}(p, x)$ by letting $y^{n}=\lambda^{n} y+\left(1-\lambda^{n}\right) \underline{u}$ for suitably chosen weights $\lambda^{n} \in[0,1]$. Indeed, if $p^{n} \cdot y(c) \leq 0$ for all $c \in C$ let $\lambda^{n}=1$, and if $p^{n} \cdot y(d)>0$ for some $d \in C$ let $\lambda^{n}$ be such that $\max _{c \in C} p^{n}\left(\lambda^{n} y(c)+\left(1-\lambda^{n}\right) \underline{u}\right)=0$. Clearly, due to the convexity of $\cap_{c \in C} \widetilde{X}_{c}$, we have that $y^{n} \in \cap_{c \in C} \widetilde{X}_{c}$ and hence $\left(q, y^{n}\right) \in \cap_{c \in C} \beta_{c}(p, x)$. As $\left(q, y^{n}\right) \in \beta_{0}\left(p^{n}, x^{n}\right)$, we obtain $\left(q, y^{n}\right) \in \beta\left(p^{n}, x^{n}\right)$. Moreover, as $p^{n}$ converges to $p$ and $p \cdot y(c) \leq 0$ for all $c \in C$, we have $\lambda^{n}$ converging to 1 , and $y^{n}$ converging to $y$, accordingly. Hence, the lower hemi continuity of $\beta$ is obtained.

The condition (c) for all $a \in C$ follows directly from assumption (ii); and for $a=0$ it is trivially satisfied by the definition of $\mathcal{P}_{0}$.

The condition (d) is fulfilled for all agents $c \in C$ by assumption (iv), and by the definition of $e_{0}$ for agent $a=0$.

To show that $(\mathrm{e})$ is satisfied for all agents, take any $(p, x) \in X$. Consider first the agent $a=0$ and any $(q, y) \in \mathcal{X}$. As $e_{0}((p, x)(q, y)) \in \beta_{0}((p, x))$ iff $q \in \Delta$ the condition (e) is satisfied for $a=0$. Now consider $c \in C$ and $(q, y) \in \mathcal{X}$ such that $e_{c}((p, x)(q, y)) \in \beta_{c}(p, x)$. As $e_{c}((p, x)(q, y))(c)=y(c)$ by assumption (iv), we have $p y(c) \leq 0$. Moreover, as $(q, y) \in \mathcal{X}$, we obtain $(q, y) \in \beta_{c}(p, x)$. Thus (e) is satisfied for all agents $a \in A$.

The condition (f') for agents $c \in C$ follows immediately from assumption (v) and our definition of $\mathcal{P}_{c}$. For the agent $a=0$ let $\Delta^{0}$ be any open set containing $\Delta$ and not containing 0 . Clearly, the preferences of $a=0$ can be extended to $\Delta^{0} \times \mathbb{R}^{C L}$. Hence the condition (f') is satisfied.

The condition $(\mathrm{g})$ is satisfied since we defined the coordination functions in $\Gamma$ such that no consumer coordinates with $a=0$ and the agent $a=0$ does not coordinate with any consumer.

We conclude that there exists an equilibrium state $\left(p^{*}, x^{*}\right) \in \mathcal{X}$ for the social system $\Gamma$. Hence, we have

(1) $\left(p^{*}, x^{*}\right) \in \beta\left(p^{*}, x^{*}\right)$ and

(2) $\nexists(q, y) \in X$ such that $e_{a}\left(\left(p^{*}, x^{*}\right),(q, y)\right) \neq\left(p^{*}, x^{*}\right)$ for some a $\in A$, and $e_{a}\left(\left(p^{*}, x^{*}\right),(q, y)\right) \in \mathcal{P}_{a}\left(p^{*}, x^{*}\right) \cap \beta_{a}\left(p^{*}, x^{*}\right) \quad$ for $\mathrm{a} \in g\left(\left(p^{*}, x^{*}\right),(q, y)\right)$.

We will now show that $\left(p^{*}, x^{*}\right)$ is a Walras equilibrium with coordination in the original economy $\mathcal{E}$. Clearly, by the definition of $\mathcal{X}$ we have that $\left(p^{*}, x^{*}\right) \in \Delta \times$ $\cap_{c \in C} X_{c}$. Hence, to show that $x^{*} \in F(\mathcal{E})$, we only need to show that $\sum_{c \in C} x^{*}(c) \leq$ 
0 . First, note that the definition of $\beta$ and condition (i) yields $p^{*} \cdot x(c) \leq 0$ for all $c \in C$ and hence $p^{*} \sum_{c \in C} x^{*}(c) \leq 0$. Now assume that there exists $h \in L$ such that $\sum_{c \in C} x_{h}^{*}(c)>0$. Define $q \in \Delta$ by $q_{k}=0$ for $k \neq h$ and $q_{h}=1$ and consider the state $\left(q, x^{*}\right) \in X$. Clearly, $p^{*} \neq q$ as $p^{*} \sum_{c \in C} x^{*}(c) \leq 0$ and $q \sum_{c \in C} x^{*}(c)>0$. Moreover, $e_{c}\left(\left(p^{*}, x^{*}\right),\left(q, x^{*}\right)\right)=\left(p^{*}, x^{*}\right)$ for all $c \in C$ and $e_{0}\left(\left(p^{*}, x^{*}\right),\left(q, x^{*}\right)\right)=$ $\left(q, x^{*}\right)$ and hence $g\left(\left(p^{*}, x^{*}\right)\left(q, x^{*}\right)\right)=\{0\}$. As $\left(\left(p^{*}, x^{*}\right),\left(q, x^{*}\right)\right) \in \mathcal{P}_{0}\left(p^{*}, x^{*}\right) \cap$ $\beta_{0}\left(p^{*}, x^{*}\right)$, we have a contradiction to $(2)$. Thus, $\sum_{c \in C} x^{*}(c) \leq 0$ and hence $x^{*} \in F(\mathcal{E})$.

Clearly, condition (I) in the definition of a Walras equilibrium with coordination is also fulfilled by the definition of $\beta_{c}, c \in C$, and (1).

To prove that (II) is satisfied, assume, conversely, that there exists a state $y \in Y$ with $y \neq x^{*}$ such that $p^{*} \cdot y(c) \leq 0$ and $e_{c}\left(x^{*}, y\right) \in X_{c} \cap \mathcal{P}_{c}\left(x^{*}\right)$ for all $c \in g\left(x^{*}, y\right)$. First, we prove that $y \in \cap_{c \in C} X_{c}$. Indeed, consider $c \in g\left(x^{*}, y\right)$. As $x^{*} \in \cap_{c \in C} X_{c}$ and $e_{c}\left(x^{*}, y\right) \in X_{c}$, assumption (v) yields $y \in X_{c}$. For $c \notin g\left(x^{*}, y\right)$ we have $e_{c}\left(x^{*}, y\right)=x^{*} \in X_{c}$ and hence, again by assumption $(\mathrm{v})$, that $y \in X_{c}$. Hence $x \in \cap_{c \in C} X_{c}$. Moreover, as $x^{*} \in F(\mathcal{E}) \subset$ int $\widetilde{Y}$ and $\cap_{c \in C} X_{c}$ is convex, we obtain that $\lambda y+(1-\lambda) x^{*} \in \cap_{c \in C} \widetilde{X}_{c}$ for $\lambda>0$ sufficiently small, say, for $\lambda=\tilde{\lambda}>0$. Let $y^{\prime}=\tilde{\lambda} y+(1-\tilde{\lambda}) x^{*}$. Then $\left(p^{*}, y^{\prime}\right) \in X$. We will now show that the state $\left(p^{*}, y^{\prime}\right)$ can be used to violate condition (2) above. Clearly, $e_{a}\left(\left(p^{*}, x^{*}\right),\left(p^{*}, y^{\prime}\right)\right) \neq\left(p^{*}, x^{*}\right)$ for $a \in g\left(x^{*}, y\right)$. Moreover, $g\left(\left(p^{*}, x^{*}\right),\left(p^{*}, y^{\prime}\right)\right)=g\left(x^{*}, y\right)$ as $0 \notin g\left(\left(p^{*}, x^{*}\right),\left(p^{*}, y^{\prime}\right)\right)$. Consider $c \in g\left(x^{*}, y\right)$. As $p^{*} y^{\prime}(c) \leq 0$, we have $e_{c}\left(\left(p^{*}, x^{*}\right),\left(p^{*}, y^{\prime}\right)\right) \in \beta_{c}\left(p^{*}, x^{*}\right)$. To show that $e_{c}\left(\left(p^{*}, x^{*}\right),\left(p^{*}, y^{\prime}\right)\right) \in \mathcal{P}_{c}\left(p^{*}, x^{*}\right)$, note that assumption (iii) implies that $\left(p^{*}, x^{*}\right)$ belongs to the boundary of $\mathcal{P}_{c}\left(p^{*}, x^{*}\right)$. Hence, the convexity of $\mathcal{P}_{c}\left(x^{*}\right)$ and $X_{c}$ implies that $\tilde{\lambda} e_{c}\left(\left(p^{*}, x^{*}\right),\left(p^{*}, y\right)\right)+(1-\tilde{\lambda})\left(p^{*}, x^{*}\right) \in \mathcal{P}_{c}\left(p^{*}, x^{*}\right)$. As $\tilde{\lambda} e_{c}\left(\left(p^{*}, x^{*}\right),\left(p^{*}, y\right)\right)+(1-\tilde{\lambda})\left(p^{*}, x^{*}\right)=e_{c}\left(\left(p^{*}, x^{*}\right),\left(p^{*}, y^{\prime}\right)\right)$, we have a violation of $(2)$.

We will now show that $p^{*} \cdot \sum_{c \in C} x^{*}(c)=0$. Assume that there exists a consumer $c \in C$ for which $p^{*} \cdot x^{*}(c)<0$. Again, by assumption (iii) and $F(\mathcal{E}) \subset$ int $\widetilde{X}$, consumer $c$ can find a net trade $y(c)$ with $p^{*} \cdot y(c)<0$ such that when $c$ is the only consumer who changes net trade, we obtain a state $\left(p^{*}, y\right) \in \mathcal{X}$ with $\left(p^{*}, y\right) \in \mathcal{P}_{c}\left(x^{*}, x^{*}\right) \cap \beta_{c}\left(p^{*}, x^{*}\right)$ for all $c \in g\left(\left(p^{*}, x^{*}\right),\left(p^{*}, y\right)\right)$. This contradicts the fact that $\left(p^{*}, x^{*}\right)$ satisfies condition (2) above. Hence condition (III) in the definition of a Walras equilibrium with coordination is satisfied.

We have now shown that $\left(p^{*}, x^{*}\right)$ is a Walras equilibrium with coordination in the economy $\mathcal{E}$.

We have already commented on the assumptions (iii), (iv), and (vii) on the coordination functions and the approval function in Theorem 1. The assumptions (i) and (ii) are well known from the existence theorems for an ordinary Walras equilibrium. However, since we allow externalities in the feasible net trade sets, we assume that 0 is in the interior of the set of feasible net trade states $X_{c}$ for 
all $c \in C$. In section 2 we discussed the feasible net trade assuption and gave two examples of sets $X_{c}, c \in C$ such that the assumption was satisfied. In both cases we obtain $0 \in \operatorname{int} X_{c}$, for all $c \in C$, if 0 is in the interior of their individually feasible net trade sets $X_{c}^{\prime}$ for all $c \in C$. The assumption that $0 \in \operatorname{int} X_{c}$ can, of course, be replaced by the assumption that $0 \in X_{c}$ by introducing the concept of a quasi Walras equilibrium with coordination and only proving the existence of such equilibria. The assumption (vi) states that the preferences of all consumers are well behaved on the boundary of their feasible net trade sets.

\section{$5 \quad$ Special cases}

In this section we consider some examples of coordination functions and approval functions. We assume that the coordination assumption is satisfied. Moreover, we assume that if a consumer $c \in C$ coordinates with a consumer $d \in C$ then he coordinates with $d$ in all commodities $h \in L$. In this case an alternative way to describe the coordination function $e_{c}$ is to specify a coordination relation $R$ on $C \times C$, where $c R d$ iff $c$ coordinates with $d$. By assumption $R$ is reflexive. Let $R_{c}=\{d \in C \mid c R d\}$ be the consumers with whom $c$ coordinates. Thus, $e_{c}(x, y)=\left((y(d))_{d \in R_{c}},(x(d))_{d \notin R_{c}}\right)$.

We say that coordination is symmetric iff $R$ is symmetric. Also, we say that coordination is transitive iff $R$ is transitive. Given a coordination relation $R$, an approval function $g: Y \times Y \rightarrow Y$ can be generated in the following way. Consider for each $c \in C$ the set $R_{c}^{-1}=\{d \in C \mid d R c\} . R_{c}^{-1}$ are all consumers $d$ who coordinate their net trade with $c$. The approval function $g$ used in Theorem 1 is given by $g(x, y)=\cup_{\{c \in C \mid x(c) \neq y(c)\}} R_{c}^{-1}$ for all $x, y \in Y$. Clearly, we could, in general, have defined an approval structure as a family $\left(B_{c}\right)_{c \in C}$ based on the interpretation that a state in which consumer $c$ has changed his net trade has to be approved by consumers in $B_{c}$. Naturally, $c \in B_{c}$ for all $c$. Moreover, we assume $B_{c} \subset R_{c}^{-1}$ because only these consumers coordinate their net trade with $c$ and are therefore informed about $c$ 's new net trade. Consequently, we define an approval structure as a family $\left(B_{c}\right)_{c \in C}$ with $\{c\} \subset B_{c} \subset R_{c}^{-1}$ for all $c \in C$. The approval function $g$ corresponding to the approval structure $\left(B_{c}\right)_{c \in C}$ is defined by $g(x, y)=\cup_{\{c \in C \mid x(c) \neq y(c)\}} B_{c}$ for all $x, y \in Y$.

\subsection{No coordination}

No coordination means that the coordination is given by the coordination relation $R$ where $c R d$ iff $c=d$. Thus, for any state $x$ and alternative state $y$, consumer c believes that the state in which he is the only one who has changed his net trade from $x(c)$ to $y(c)$ will be the outcome. As $c \in B_{c} \subset R_{c}^{-1}$, the approval structure must be given by $B_{c}=\{c\}$. Clearly, condition (II) in the definition of a Walras equilibrium with coordination in this case states that there is no consumer $c$ who 
can find a $y(c) \in \mathbb{R}^{L}$ with $p y(c) \leq 0$ such that $\left((x(d))_{d \neq c}, y(c)\right) \in \mathcal{P}_{c}(x)$. Thus, if there is no coordination in $\mathcal{E}$, a Walras equilibrium with coordination is indeed a traditional Walras equilibrium.

\subsection{Coordination in a partition}

Remark 1. Coordination according to the relation $R$ is symmetric and transitive if and only if the family $\left(R_{c}\right)_{c \in C}$ is a partition of $C$.

Coordination in a partition can be used to model the decision problem in an economy with households. Typically, a household consists of several consumers whose preferences are interdependent. Hence let us assume that the set of consumers is divided into households and that the coordination relation is given by coordination in the partition of households. We still have to specify the approval structure. Assume that the approval structure is the one considered in Theorem 1, i.e. $B_{c}=R_{c}^{-1}=R_{c}$ for all $c \in C$. Condition (I) in the definition of a Walras equilibrium with coordination in this economy states that each member of a household individually has to satisfy the budget constraint. Condition (II) states that there is no households whose members can find new net trades satisfying their budget constraints such that all members of the household prefer the state in which they obtain the new net trades. For example, it is impossible to improve the situation of the members of a household by their simultaneously stopping smoking or starting drinking. Note that the Walras equilibria with coordination in this economy are exactly the same as the ones in the economy where all members of a household have identical preferences given by the intersection of the preference correspondences of the household members. Indeed, when there is coordination in a partition, then $e_{d}(x, y)=e_{c}(x, y)$ for all $d, c$ in the same element of the partition. Hence, condition II in the definition of a Walras equilibrium with coordination is satisfied iff there does not exist an element $F$ in the partition and net trades $(y(c))_{c \in F}$ with the property that $p y(c) \leq 0$ and $e_{c}(x, y) \in \cap_{d \in F} \mathcal{P}_{d}(x)$ for all $c \in F$.

Clearly, the equilibrium concept becomes weaker if a given approval structure is enlarged. The existence theorem in this paper assumes that $B_{c}=R_{c}^{-1}=R_{c}$, that is, the largest possible approval structure is assumed. However, it should be noted that if we had considered approval structures $\left(B_{c}^{\prime}\right)$ such that $B_{c}^{\prime} \subset B_{c}$, then there would not necessarily exist a Walras equilibrium with coordination. Consider, for example, an economy with two consumers $C=\{1,2\}$ and two commodities, and the coordination relation $i R j$ for $i, j \in C$. Let $X_{1}=X_{2}=$ $X=\left(\mathbb{R}_{+}^{2}-\{\omega\}\right) \times\left(\mathbb{R}_{+}^{2}-\{\omega\}\right)$ for some $\omega \in \mathbb{R}_{+}^{2}$ and let the consumers preference correspondences be given by $u_{i}: X \rightarrow X$ with $\operatorname{grad}_{x_{j}} u_{i} \neq 0$ for all $i, j \in C$. When the approval structure is $B_{1}=\{1\}$ and $B_{2}=\{2\}$, we can easily choose $u_{1}$ and $u_{2}$ such that the first order conditions for a Walras equilibrium with coordination are inconsistent. 


\subsection{Complete coordination}

Complete coordination is the coordination relation $R$ for which $c R d$ for all $c, d \in$ $C$ and approval structure $B_{c}=C$ for all $c \in C$. Hence for all $c \in C$ the coordination function is given by $e_{c}(x, y)=y$, and the approval function is given by $g(x, y)=C$ for all $x, y \in Y$. Note, that even with complete coordination, Walras equilibria with coordination is not, in general, Pareto optimal. The reason is that consumers have individual budget constraints, and without independence some transfer of budgets might be advantageous for all agents.

\subsection{Arbitrary coordination structures}

Clearly, there are many interpretations where coordination structures that are not symmetric or transitive are natural. In a hierarchical structure, or when consumers choose sequentially, coordination is not symmetric. Moreover, assume that we have households and coordination within households, but that, in addition, consumers have partner relations with other consumers, creating further coordination for some consumers. In this case, we will typically have a situation in which coordination is not transitive.

In general there is no trivial connection between Walras equilibria in an economy and Walras equilibria with coordination when the description of the basic economy has been enlarged with a coordination relation $R$ and an approval structure $\left(B_{c}\right)_{c \in C}$. Consider, however, the case where $B_{c}=\{c\}$ for all $c \in C$ and hence $g(x, y)=\{c \in C \mid x(c) \neq y(c)\}$. Clearly, here the set of equilibria with coordination is a subset (possibly empty) of the set of Walras equilibria. Coordination might, also enlarge the set of equilibria, though ${ }^{3}$. Consider again an economy with $C=\{1,2\}$. Let $X_{1}=\left(\mathbb{R}_{+}^{L}-\left\{\omega_{1}\right\}\right) \times \mathbb{R}^{L}$ and $X_{2}=\left(\mathbb{R}_{+}^{L}-\left\{\omega_{1}\right\}\right) \times\left(\mathbb{R}_{+}^{L}-\left\{\omega_{2}\right\}\right)$ with $\omega_{i} \in \mathbb{R}_{+}^{L}$ for $i=1,2$. Let the consumers' preference correspondences be given by the utility functions $u_{1}: X_{1} \rightarrow \mathbb{R}$ with $u_{1}(x(1), x(2))=v_{1}(x(1))$ for some strictly monotone function $v_{1}: \mathbb{R}_{+}^{L}-\left\{\omega_{1}\right\} \rightarrow \mathbb{R}$, and $u_{2}: X_{2} \rightarrow \mathbb{R}$ with $u_{2}(x(1), x(2))=v_{2}(x(2))-v_{1}(x(1))$ for some strictly monotone function $v_{2}: \mathbb{R}_{+}^{L}-\left\{\omega_{2}\right\} \rightarrow \mathbb{R}$. Assume that $R_{1}=\{1\}$ and $R_{2}=\{1,2\}$ and that $B_{c}=R_{c}^{-1}$ for $c \in C$. Hence $B_{1}=\{1,2\}$ and $B_{2}=\{2\}$. Clearly, in this example, the set of Walras equilibria is a subset of the Walras equilibria with coordination. However, the set of Walras equilibria with coordination is much larger than the set of Walras equilibria. We will show that $\left(p^{*}, x^{*}\right) \in \Delta \times Y$ is a Walras equilibrium with coordination if $x^{*}(2)$ is a maximal element for $v_{2}$ in $\left\{x \in \mathbb{R}_{+}^{L}-\left\{\omega_{2}\right\} \mid p^{*} x \leq 0\right\}$ and $x^{*}(1)=-x^{*}(2) \in \mathbb{R}_{+}^{L}-\left\{\omega_{1}\right\}$. Clearly, $x^{*}$ is feasible. Moreover, as $v_{2}$ is strictly monotone, we have $p^{*} x^{*}(2)=0$ and hence $p^{*} x^{*}(1)=0$. Thus, condition I is satisfied. Condition III is trivially satisfied as $x^{*}(1)+x^{*}(2)=0$. Now assume that condition II is violated. Hence there exists

\footnotetext{
${ }^{3}$ It can easily be shown that, in general, this holds with the type of preferences used in the next example.
} 
$y \neq x^{*}$ such that $p^{*} y(c) \leq 0$ and $e_{c}\left(x^{*}, y\right) \in \mathcal{P}_{c}$ for $c \in g\left(x^{*}, y\right)$. First, assume that $y(2)=x^{*}(2)$. Hence $g\left(x^{*}, y\right)=B_{1}=\{1,2\}$. However, by definition of $x^{*}(2)$ we cannot have $e_{1}\left(x^{*}, y\right) \in \mathcal{P}_{1}\left(x^{*}\right)$ and $e_{2}\left(x^{*}, y\right) \in \mathcal{P}_{2}\left(x^{*}\right)$. Second, assume that $y(1)=x^{*}(1)$. Thus, $g\left(x^{*}, y\right)=B_{2}=\{2\}$ and again the definition of $x^{*}(2)$ results in a contradiction. Last, consider the case where $y(1) \neq x^{*}(1)$ and $y(2) \neq x^{*}(2)$. Then, as $p^{*} y(1) \leq 0, p^{*} y(2) \leq 0$, and $g\left(x^{*}, y\right)=\{1,2\}$, the definition of $x^{*}(2)$ again implies that it is impossible to have $e_{1}\left(x^{*}, y\right)=\left(y(1), x^{*}(2)\right) \in \mathcal{P}_{1}\left(x^{*}\right)$ and $e_{2}\left(x^{*}, y\right)=y \in \mathcal{P}_{2}\left(x^{*}\right)$. Again we have a contradiction. Hence, we conclude that $\left(p^{*}, x^{*}\right)$ is a Walras equilibrium with coordination. Note, that if, in the example, we use the approval structure $B_{c}=\{c\}$ for $c \in C$, then the set of Walras equilibria with coordination is identical to the set of Walras equilibria.

\section{Appendix: A Social System with Coordina- tion}

This section repeats concepts from Vind (1983) and repeats the existence theorem for an equilibrium in Vind (1983) with the assumptions in the improved version in Keiding (1985).

A social system with coordination is an indexed family

$$
\Gamma=\left(A,\left(\mathcal{X}_{a}, \beta_{a}, \mathcal{P}_{a}, e_{a}\right)_{a \in A}, g\right)
$$

where

- $A$ is a finite set of agents,

- $X_{a} \subset \mathbb{R}^{\ell}$ are the feasible states for agent $a$. The state space $\mathcal{X}$ of the system is defined as $\mathcal{X}=\cap_{a \in A} \mathcal{X}_{a}$,

- $\beta_{a}: X \rightarrow 2^{X}$ gives for each $x \in X$, the set, $\beta_{a}(x)$, of states which are attainable for agent $a$. The correspondence $\beta: X \rightarrow 2^{X}$ is defined as $\beta(x)=$ $\cap_{a \in A} \beta_{a}(x)$,

- $\mathcal{P}_{a}: X \rightarrow 2^{X}$ is the preference correspondence of agent $a \in A$,

- $e_{a}: X \times X \rightarrow X$ is the coordination function of $a \in A$,

- $g: X \times X \rightarrow 2^{A}$ is the approval function.

Definition (Equilibrium). An equilibrium in the social system with coordination is a state $x^{*} \in \mathcal{X}$ such that

(1) $x^{*} \in \beta\left(x^{*}\right)$ and

(2) $\nexists y \in X$ such that $e_{a}\left(x^{*}, y\right) \neq x^{*}$ for some $a \in A$, and $e_{a}\left(x^{*}, y\right) \in$ $\mathcal{P}_{a}\left(x^{*}\right) \cap \beta_{a}(x)$ for $a \in g\left(x^{*}, y\right)$. 
Theorem 2. Let $\left(A, \mathcal{X},\left(\beta_{a}, \mathcal{P}_{a}, e_{a}\right)_{a \in A}, g\right)$ be a social system with coordination such that for all $a \in A$ and for all $x \in \mathcal{X}$

(a) $X$ is a non-empty, convex, compact subset of $\mathbb{R}^{\ell}$,

(b) $\beta$ is continuous with closed, convex and non-empty values,

(c) $\mathcal{P}_{a}$ has open graph, convex values and $x \notin \mathcal{P}_{a}(x)$,

(d) $e_{a}$ is continuous, $e_{a}(x, \cdot)$ is affine, and $e_{a}(x, x)=x$,

(e) $e_{a}(x, y) \in \beta_{a}(x) \Rightarrow y \in \beta_{a}(x)$,

(f') there exists a convex and compact set $\mathcal{X}^{a}$ with $\mathcal{X} \subset$ int $\mathcal{X}^{a}$ and a correspondence $\mathcal{P}^{a}: X^{a} \rightarrow X^{a}$ which is irreflexive with open graph and convex (possibly empty) values such that $\mathcal{P}^{a}(x)=\mathcal{P}_{a}(x) \cap \mathcal{X}$ for all $x \in \mathcal{X}$,

(g) $g(x, y)=\left\{a \in A \mid e_{a}(x, y) \neq x\right\}$.

Then there exists an equilibrium in $\Gamma$.

Proof. See Corollary 1 in Keiding $(1985)^{4}$.

\section{References}

Arrow, K., G. Debreu: Existence of an Equilibrium for a Competitive Economy, Econometrica 22, 265-290 (1954)

Browning, M., P.A. Chiappori: Efficient Intra-Household Allocations: A General Characterization and Empirical Tests, Econometrica 66, 1241-1278 (1998)

Duffie, D., W. Shafer: Equilibrium in Incomplete Markets: I; A Basic Model of Generic Existence, Journal of Mathematical Economics 14, 285-300 (1985)

Gersbach, H., H. Haller: Intra-Household Bargaining Power and Equilibrium Consumption, Working Paper Virginia Tech 99-03, (1999)

Grodal, B., K. Vind: Equilibrium with Arbitrary Market Structures, University of Copenhagen, (1989)

Keiding, H.: On the Existence of Equilibrium in Social Systems with Coordination, Journal of Mathematical Economics 14, 105-111 (1985)

Shafer,W., H. Sonnenschein: Equilibrium in Abstract Economies without Ordered Preferences, Journal of Mathematical Economic 2, 345-348 (1975)

Vind, K.: Equilibrium with Coordination, Journal of Mathematical Economics $12,275-285(1983)$

\footnotetext{
${ }^{4}$ The assumption that $\beta$ has convex values has been forgotten in the statement of Corollary 1 in Keiding (1985).
} 